\title{
Oncogenic UBE3C promotes breast cancer progression by activating $\mathrm{Wnt} / \beta$-catenin signaling
}

Chen Hang ${ }^{1 \dagger}$, Shanojie Zhao ${ }^{1+}$, Tiejun Wang ${ }^{1}$ and Yan Zhang ${ }^{1,2^{*}}$

\begin{abstract}
Background: Breast cancer ( $\mathrm{BrCa}$ ) is the most common female malignancy worldwide and has the highest morbidity among all cancers in females. Unfortunately, the mechanisms of BrCa growth and metastasis, which lead to a poor prognosis in BrCa patients, have not been well characterized.

Methods: Immunohistochemistry (IHC) was performed on a BrCa tissue microarray (TMA) containing 80 samples to evaluate ubiquitin protein ligase E3C (UBE3C) expression. In addition, a series of cellular experiments were conducted to reveal the role of UBE3C in BrCa.

Results: In this research, we identified UBE3C as an oncogenic factor in BrCa growth and metastasis for the first time. UBE3C expression was upregulated in BrCa tissues compared with adjacent breast tissues. BrCa patients with high nuclear UBE3C expression in tumors showed remarkably worse overall survival (OS) than those with low nuclear expression. Knockdown of UBE3C expression in MCF-7 and MDA-MB-453 BrCa cells inhibited cell proliferation, migration and invasion in vitro, while overexpression of UBE3C in these cells exerted the opposite effects. Moreover, UBE3C promoted $\beta$-catenin nuclear accumulation, leading to the activation of the $\mathrm{Wnt} / \beta$-catenin signaling pathway in $\mathrm{BrCa}$ cells.
\end{abstract}

Conclusion: Collectively, these results imply that UBE3C plays crucial roles in BrCa development and progression and that UBE3C may be a novel target for the prevention and treatment of $\mathrm{BrCa}$.

Keywords: Breast cancer, UBE3C, $\beta$-catenin, Cancer progression

\section{Background}

Breast cancer $(\mathrm{BrCa})$ has the highest morbidity among all cancers in females worldwide and may have caused more than 40,000 cancer-related deaths in the United States in 2019 [1]. Current therapeutic approaches for $\mathrm{BrCa}$ mainly focus on comprehensive treatment, including surgery, chemotherapy, radiotherapy, hormone therapy, and targeted therapy $[2,3]$. $\mathrm{BrCa}$ in situ is usually not fatal to

\footnotetext{
*Correspondence: fuyou2007@126.com

${ }^{\dagger}$ Chen Hang and Shanojie Zhao contributed equally to this work

1 Department of Oncology, Wuxi Maternal and Child Health Hospital Affiliated to Nanjing Medical University, Wuxi 214023, China

Full list of author information is available at the end of the article
}

patients; however, advanced $\mathrm{BrCa}$ with lymph node and/ or distant metastasis tends to cause life-threatening outcomes for patients [4]. Although increasing numbers of biomarkers and potential therapeutic targets for $\mathrm{BrCa}$ have been preliminarily explored, there is no defined means for diagnosis and prognostic assessment of $\mathrm{BrCa}$ $[5,6]$. Thus, further exploration of novel biomarkers and the potential mechanisms of $\mathrm{BrCa}$ is still needed.

Ubiquitin protein ligase E3C (UBE3C), also named HECTH2, is an important regulator of proteasome function that continuously cycles on and off proteasomes and stimulates associations by ubiquitin conjugates through cooperation with USP14 [7]. UBE3C has been defined as a significant cancer-related functional protein that

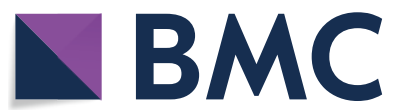

(c) The Author(s) 2021. This article is licensed under a Creative Commons Attribution 4.0 International License, which permits use, sharing, adaptation, distribution and reproduction in any medium or format, as long as you give appropriate credit to the original author(s) and the source, provide a link to the Creative Commons licence, and indicate if changes were made. The images or other third party material in this article are included in the article's Creative Commons licence, unless indicated otherwise in a credit line to the material. If material is not included in the article's Creative Commons licence and your intended use is not permitted by statutory regulation or exceeds the permitted use, you will need to obtain permission directly from the copyright holder. To view a copy of this licence, visit http://creativeco mmons.org/licenses/by/4.0/. The Creative Commons Public Domain Dedication waiver (http://creativecommons.org/publicdomain/ zero/1.0/) applies to the data made available in this article, unless otherwise stated in a credit line to the data. 
promotes cancer metastasis and growth in multiple cancers, including melanoma [8], renal cell carcinoma [9], and non-small cell lung cancer [10]. In $\mathrm{BrCa}, \mathrm{UBE} 3 \mathrm{C}$ was identified as a novel downstream molecule of ER $\alpha$, and estrogen remarkably stimulates the E3 activity of UBE3C on CCNB1, which promotes BrCa progression [11]. In addition, the UBE3C-mediated malignant phenotypes of BrCa can be suppressed by miR-30a-5p [12]. However, the role of $\mathrm{UBE} 3 \mathrm{C}$ in $\mathrm{BrCa}$ and its potential mechanisms have not been well characterized.

Here, we reported that UBE3C was overexpressed in $\mathrm{BrCa}$ tissues and associated with advanced clinical phenotypes and a poor prognosis. In addition, inhibition of UBE3C significantly suppressed BrCa cell proliferation, migration and invasion. Significantly, UBE3C notably promoted the activation of the $\mathrm{Wnt} / \beta$-catenin pathway by promoting $\beta$-catenin nuclear accumulation. Our research reveals mechanistic insights into the UBE3C-mediated malignant phenotypes of $\mathrm{BrCa}$, which provides a potential biomarker for the diagnosis and prognostic assessment of $\mathrm{BrCa}$.

\section{Methods}

\section{Clinical samples}

A tissue microarray (TMA) containing $80 \mathrm{BrCa}$ samples and corresponding normal tissue samples (BRC1602) was obtained from Superbiotek Inc. (Shanghai, China). Relevant clinicopathological characteristics recorded for each case were also provided by Superbiotek Inc. The use of the clinical samples in this research was approved by the Medicine Ethical Committee of Wuxi Maternal and Child Health Hospital affiliated with Nanjing Medical University.

\section{Immunohistochemistry}

Immunohistochemistry (IHC) was performed on the TMA. TMA sections were deparaffinized at $55^{\circ} \mathrm{C}$ for $30 \mathrm{~min}$. The sections were then washed with xylene for three $5 \mathrm{~min}$. The sections were rehydrated with successive washes in $100 \%, 90 \%$ and $70 \%$ gradient ethanol. Hydrogen peroxidase (0.3\%, ZSGB-Bio, Beijing, China) was applied for $20 \mathrm{~min}$ to block endogenous peroxidase activity. A primary antibody against UBE3C (1:200 dilution, Cat. ab101512, Abcam) was used to visualize UBE3C expression. Previously described quantitative evaluation criteria were used [13, 14]. Briefly, the percentage of positively stained cells was scored on a $0-4$ scale: 0 (<5\%), 1 (6-25\%), 2 (26-50\%), 3 (51-75\%) and $4(>75 \%)$. The staining intensity was scored on a $0-3$ scale: 0 (negative), 1 (weak), 2 (moderate), and 3 (strong). The immunoreactivity score (IRS) was calculated by multiplying the percentage of positive cells by the staining intensity. Immunostained sections were scanned using Aperio Digital Pathology Slide Scanners.

\section{Cell culture and transfection}

The MCF-10A, MCF-7, and MDA-MB-453 cell lines were obtained from KeyGEN BioTECH Inc. (Nanjing, China). MCF-7 and MDA-MB-453 cells were maintained in RPMI-1640 medium (KeyGEN BioTECH Inc.) supplemented with $10 \%(\mathrm{v} / \mathrm{v})$ fetal bovine serum (FBS) at $37{ }^{\circ} \mathrm{C}$ with $5 \% \mathrm{CO}_{2}$. MCF-10A cells were cultured in DMEM/F12 (KeyGEN BioTECH Inc.) supplemented with $5 \%(\mathrm{v} / \mathrm{v})$ horse serum, $20 \mathrm{ng} / \mathrm{mL}$ human EGF, $10 \mu \mathrm{g} / \mathrm{mL}$ insulin, $0.5 \mu \mathrm{g} / \mathrm{mL}$ hydrocortisone, penicillin, streptomycin and $100 \mathrm{ng} / \mathrm{mL}$ cholera toxin.

For subsequent assays, MCF-7 and MDA-MB-453 cells were transfected with UBE3C-siRNAs (The sequences of siRNAs for UBE3C were shown in Table 1), siRNA\#NC, a UBE3C plasmid (Cloning vector: pcDNA3.1(+)-EGFP), or a control plasmid, which were synthesized by KeyGEN BioTECH Inc. (Nanjing, China), using Lipofectamine 2000 Reagent (Invitrogen) according to the manufacturer's instructions.

\section{Quantitative real-time PCR}

Total RNA was extracted from BrCa cells using TRIzol reagent (Invitrogen). The primers for UBE3C mRNA reverse transcription were synthesized by KeyGEN BioTECH Inc. (Nanjing, China). qRT-PCR was conducted using the One Step TB Green ${ }^{\mathrm{TM}}$ PrimeScript $^{\mathrm{TM}}$ RT-PCR Kit II (SYBR Green, TaKaRa). The primers used for gene amplification were as follows: UBE3C: $5^{\prime}$ TGGTGG CAGACTACAGGCTGAA $3^{\prime}$ (forward), $5^{\prime}$ GAGGCT GACGACATTGGCAAGG $3^{\prime}$ (reverse); and GAPDH: $5^{\prime}$ AGATCATCAGCAATGCCTCCT $3^{\prime}$ (forward), $5^{\prime}$ TGA GTCCTTCCACGATACCAA $3^{\prime}$ (reverse). The $2^{-\Delta \Delta \mathrm{Ct}}$ method was used for mRNA expression analysis.

\section{Table 1 The sequences of siRNAs for UBE3C}

\begin{tabular}{ll}
\hline siRNAs & Sequences \\
\hline siRNA\#1 & 5'-UGAAGAAGCUGGACACAAATT-3' \\
siRNA\#2 & 5'-UUUGUGUCCAGCUUCUUCATT-3' \\
& 5'-GGAAGAAAGAAGAAAGAGATT-3' \\
siRNA\#3 & 5'-UCUCUUUCUUCUUUCUUCCTT-3' \\
& 5'-CCAUAGAAGUUGUAGGUCATT-3' \\
SiRNA\#NC & 5'-UGACCUACAACUUCUAUGGTT-3' \\
& 5'-UUCUCCGAACGUGUCACGUTT-3' \\
& 5'-ACGUGACACGUUCGGAGAATT-3' \\
\hline
\end{tabular}




\section{Western blot analysis}

Cells were placed in $35-\mathrm{mm}$ dishes $\left(6 \times 10^{5}\right.$ cells/dish). Forty-eight hours after transfection, all cells were harvested with lysis buffer for total protein extraction. SDSpolyacrylamide gel electrophoresis and western blot analysis were performed according to standard protocols. For nuclear and total protein extraction, a nuclear and total protein extraction kit was used (KeyGEN BioTECH Inc.). Primary antibodies specific for UBE3C (1:1000 dilution, Cat. ab101512, Abcam), $\beta$-catenin (1:1000 dilution, Cat. ab22656, Abcam), Cyclin D1 (1:1000 dilution, Cat. ab40754, Abcam), and MMP9 (1:1000 dilution, Cat. ab76003, Abcam) were used. The expression levels of proteins were normalized to those of GAPDH for each sample.

\section{CCK-8 assay}

Cell Counting Kit-8 (CCK-8) assay is a simple and effective method to check that capability of cell proliferation. Forty-eight hours after transfection, cells were digested using $0.25 \%$ trypsin for $1 \mathrm{~min}$ and resuspended in RPMI 1640 medium containing 10\% FBS. The suspended cells were seeded in a 96-well plate with the cell density adjusted to $4 \times 10^{4}$ cells $/ \mathrm{mL}(100 \mu \mathrm{L} /$ well $)$ and incubated at $37{ }^{\circ} \mathrm{C}$ in a constant-temperature incubator with $5 \%$ $\mathrm{CO}_{2}$ for $12,24,48$, or $78 \mathrm{~h}$. To each well, $10 \mu \mathrm{L}$ CCK-8 was added, after which the plate was placed in the incubator for $2 \mathrm{~h}$. The OD value of each well was measured at $450 \mathrm{~nm}$ with a microplate reader. Each experiment was repeated three times.

\section{Wound healing assay}

For wound healing analysis, $\mathrm{BrCa}$ cells were seeded in 96-well plates (Costar, Corning, NY) and cultured to confluence. The cell monolayers were wounded by removing the culture insert and rinsed with PBS to remove cell debris. After $6 \mathrm{~h}$ of migration, the cells were stained with $0.2 \%(\mathrm{v} / \mathrm{v})$ crystal violet for $20 \mathrm{~min}$ at room temperature. Images were acquired at $0 \mathrm{~h}$ and $6 \mathrm{~h}$ after wounding using a Nikon optics microscope connected to a PowerShot G10 camera (Canon, Tokyo, Japan). The migratory area was calculated by subtracting the distance between the edges of the wound at $6 \mathrm{~h}$ from that at $0 \mathrm{~h}$.

\section{Boyden chamber assay}

For cell invasion assays, $5 \times 10^{4}$ cells in serum-free medium supplemented with $5 \mathrm{mg} / \mathrm{mL}$ BSA were inoculated into the upper compartments of a modified Boyden chamber $(8.0 \mu \mathrm{m}$, Costar, Corning, NY). The polycarbonate membranes of the Boyden chambers were coated with Matrigel (BD Biosciences, Franklin Lakes, NJ). After $6 \mathrm{~h}$, the invasive cells on the lower side of the Boyden chambers were fixed and stained with $0.2 \%$ crystal violet. The stained cells were imaged, and five microscopic fields per sample were randomly selected for quantification.

\section{Immunofluorescence}

Coverslips were immersed in cell medium to allow cells to attach and grow, and then they were washed with PBS three times for 5 min each time. Paraformaldehyde (4\%) was applied to fix the cells on the coverslips for $15 \mathrm{~min}$ at room temperature. The coverslips were washed with PBS three times for 3 min each time. Then, the coverslips were incubated with PBS containing 0.5\% Triton X-100 for 5 min. Next, the cells were blocked using 5\% skim milk for $1 \mathrm{~h}$, after which anti- $\beta$-catenin antibody (1:200 dilution, Cat. ab22656, Abcam) and anti-UBE3C antibody (1:100 dilution, Cat. A6442, Abclonal) were added. After incubation overnight at $4{ }^{\circ} \mathrm{C}$, the coverslips were washed with PBS once and incubated with corresponding second antibodies (Goat anti mouse IgG-FITC, Goat anti rabbit IgG-TRITC, 1:100 dilution, KeyGEN BioTECH Inc.) at room temperature for $1 \mathrm{~h}$. Next, the coverslips were washed with PBS and stained using DAPI. After washing with PBS, the coverslips were sealed with ProLong ${ }^{\mathrm{TM}}$ Live Antifade Reagent (P36974, Thermo Fisher, USA.). Finally, the cells were observed under a fluorescence microscope.

\section{Statistical analysis}

All statistical analyses were performed using SPSS 26.0 software (Chicago, IL). Most of the data were analyzed by Student's t-test or one-way ANOVA followed by Dunnett's multiple post hoc tests. All data are presented as the means \pm SDs of five independent experiments if not otherwise noted. The associations between UBE3C expression levels and clinicopathological characteristics of $\mathrm{BrCa}$ were evaluated using Pearson's chi-squared test. All statistical tests were two-sided, and a $P$ value $\leq 0.05$ was considered statistically significant.

\section{Results \\ UBE3C is overexpressed in $\mathrm{BrCa}$}

To explore the effects of UBE3C on BrCa development, the expression of UBE3C was first assessed in $80 \mathrm{BrCa}$ and matched breast tissue samples. As shown in Fig. 1a, UBE3C was mainly located in the nucleus but was also expressed in the cytoplasm to some extent (Fig. 1a). We next evaluated the expression levels of UBE3C in the nucleus and cytoplasm in $\mathrm{BrCa}$ tissues compared with breast tissues, and the results showed that most tumor samples exhibited higher UBE3C expression in the nucleus and cytoplasm (Fig. 1b, c). In addition, compared with breast tissues, $\mathrm{BrCa}$ tissues showed overexpression of UBE3C in both the nucleus and cytoplasm when we used a paired Student's t-test (Fig. 1d, e). In addition, 

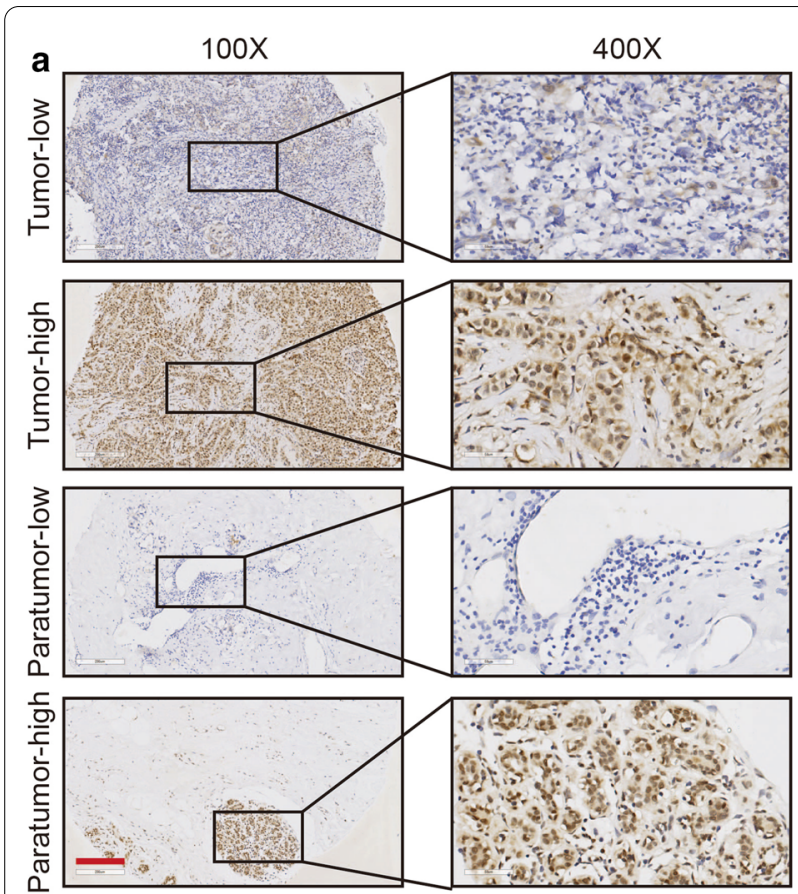

b

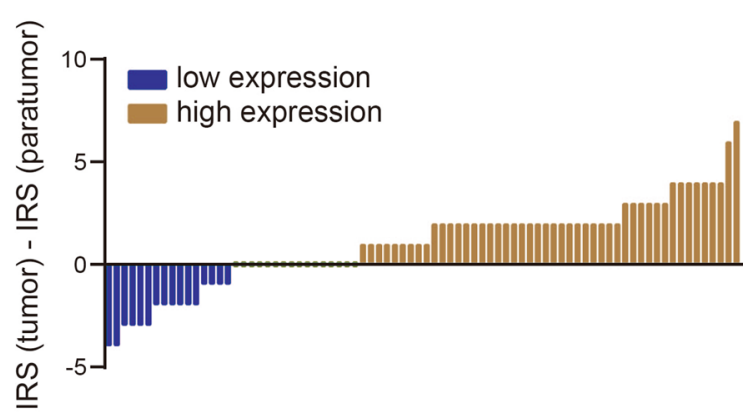

c

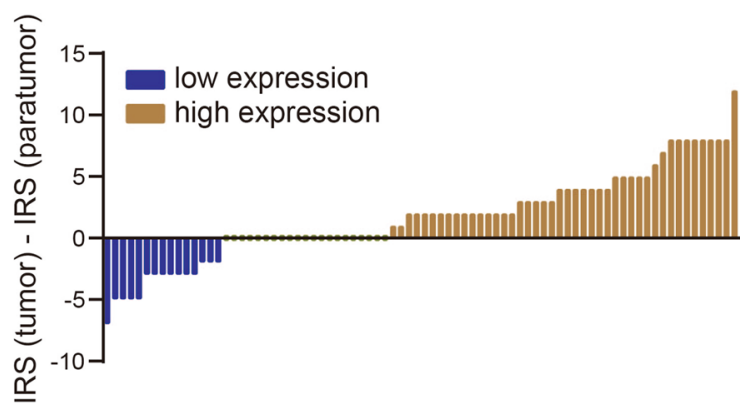

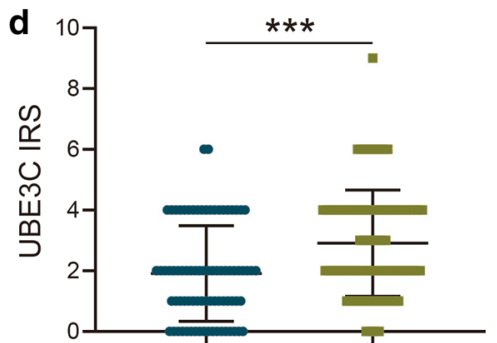

Paratumor Tumor

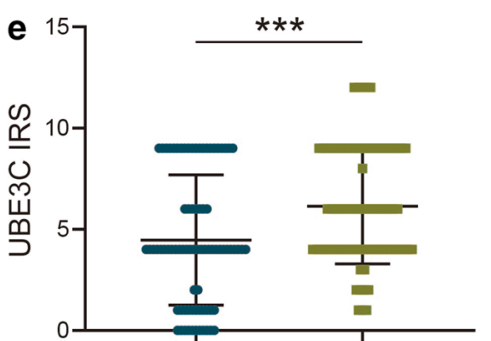

Paratumor Tumor

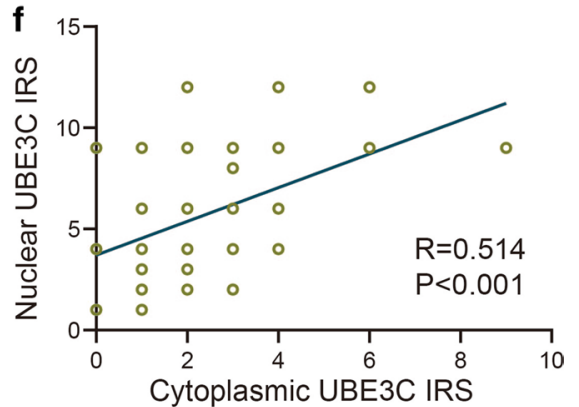

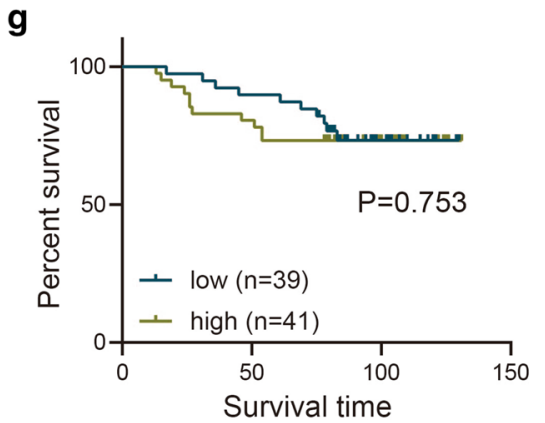

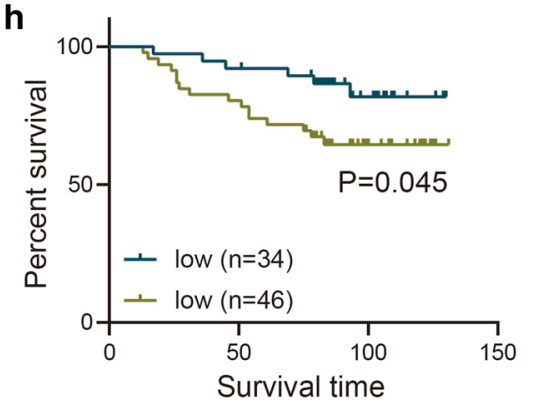

Fig. 1 UBE3C expression in BrCa tissues. a Representative images showing BrCa and paired breast tissue samples stained for UBE3C. b, c The differences in cytoplasmic and nuclear UBE3C between BrCa tissues and normal tissues. $\mathbf{d}$, e The relative expression levels of cytoplasmic and nuclear UBE3C in BrCa tissues compared with normal tissues. $\mathbf{f}$ Correlation between cytoplasmic and nuclear UBE3C. $\mathbf{g}$ Kaplan-Meier survival analysis of the OS of patients with BrCa stratified according to their cytoplasmic UBE3C expression level. $\mathbf{h}$ Kaplan-Meier survival analysis of the OS of patients with BrCa stratified according to their nuclear UBE3C expression level. ${ }^{* * P}<0.001$. Bar $=200 \mu \mathrm{m}$ 
cytoplasmic and nuclear UBE3C showed a positive correlation (Fig. 1f). Overall, similar to the expression pattern in other tumors [8-10], these results indicate that the expression of UBE3C is significantly elevated in $\mathrm{BrCa}$ and that upregulation of UBE3C may play an critical role in $\mathrm{BrCa}$ progression.

\section{UBE3C overexpression is associated with a poor prognosis in $\mathrm{BrCa}$}

We next analyzed associations between UBE3C expression and clinical parameters or prognosis in a 40-case $\mathrm{BrCa}$ cohort. High nuclear UBE3C expression was associated with an advanced tumor node metastasis classification (TNM) stage and a poor survival status (Additional file 1: Table S1). However, cytoplasmic UBE3C expression had no obvious associations with clinical parameters (Additional file 1: Table S1). Next, we evaluated the association between UBE3C expression and overall survival (OS) by Kaplan-Meier survival analysis with the log-rank test to assess significant differences. No significant differences were observed between the high (IRS $>2$ ) and low (IRS $\leq 2)$ cytoplasmic UBE3C expression groups (Fig. 1g). However, OS was significantly shortened in the high nuclear UBE3C expression group (IRS $>4$ ) compared to the low nuclear UBE3C expression group (IRS $\leq 4$ ) (Fig. 1h).

\section{Silencing UBE3C inhibits BrCa cell proliferation, migration and invasion}

Small interfering RNA (siRNA)-mediated silencing of UBE3C expression in MCF-7 and MDA-MB-453 BrCa cells was performed to assess the functional role of $\mathrm{UBE} 3 \mathrm{C}$ in $\mathrm{BrCa}$ in vitro. The functional roles of UBE3C
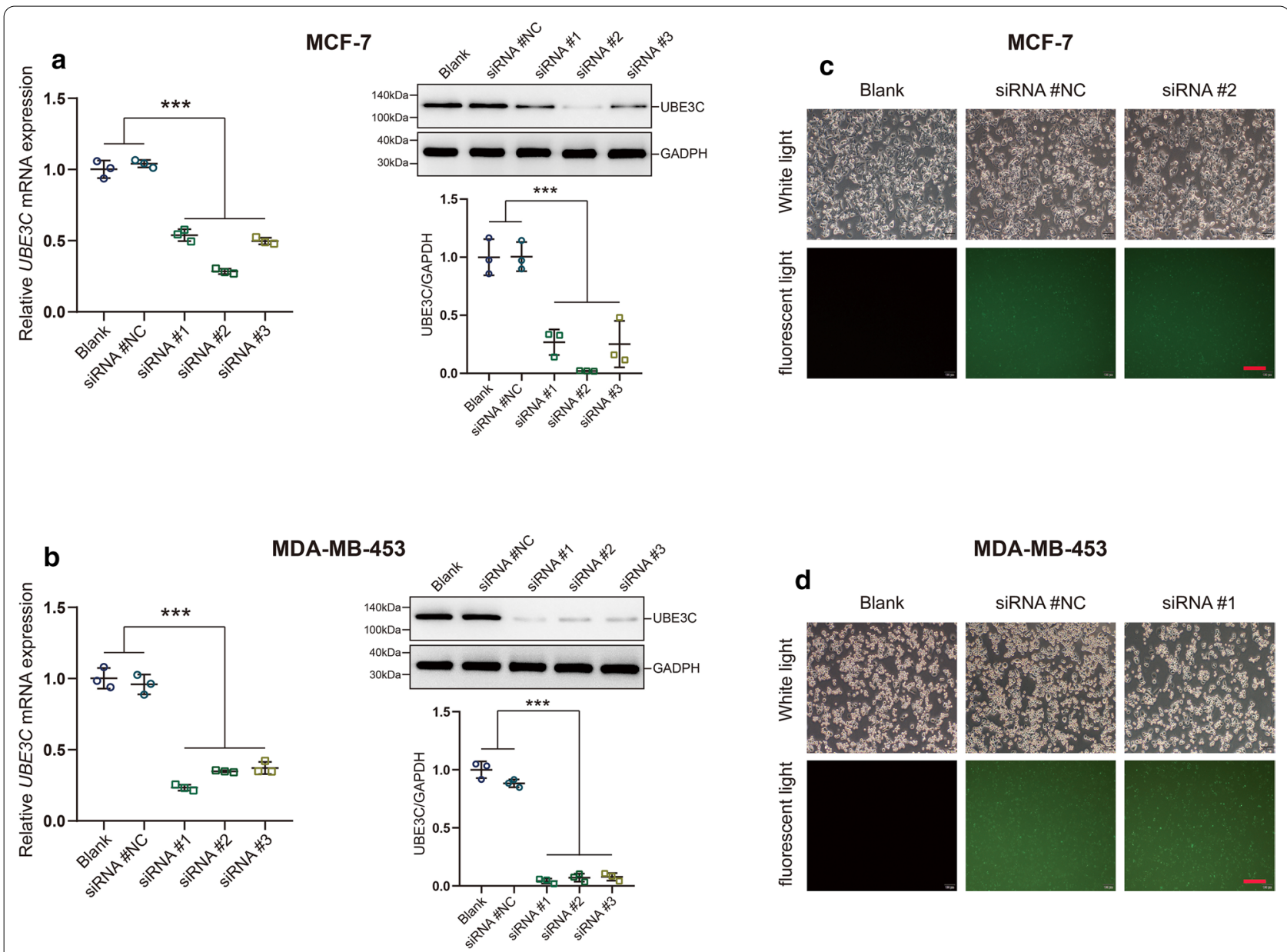

MDA-MB-453

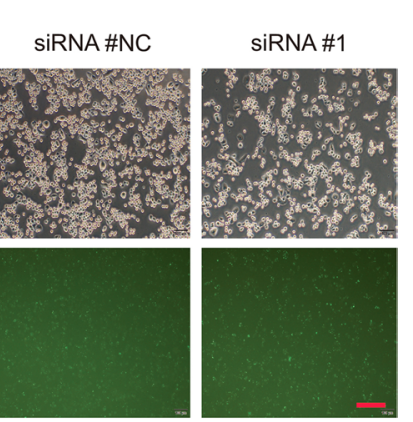

Fig. 2 The silencing efficiency of UBE3C in BrCa cells. a, b The silencing efficiency of UBE3C in BrCa cells was confirmed by qPCR and western blotting analysis. SiRNA \#2 and siRNA \#1 showed highest silencing efficiency for UBE3C in MCF-7 and MDA-MB-453 cells, respectively. ${ }^{* * * P}<0.001$. c, $\mathbf{d}$ The silencing efficiency of UBE3C in BrCa cells and cytomorphology was determined by microscopy and immunofluorescence analysis. Green fluorescence indicated that the cells have been successfully transfected. ${ }^{* *} \mathrm{P}<0.001$. Bar $=200 \mu \mathrm{m}$ 


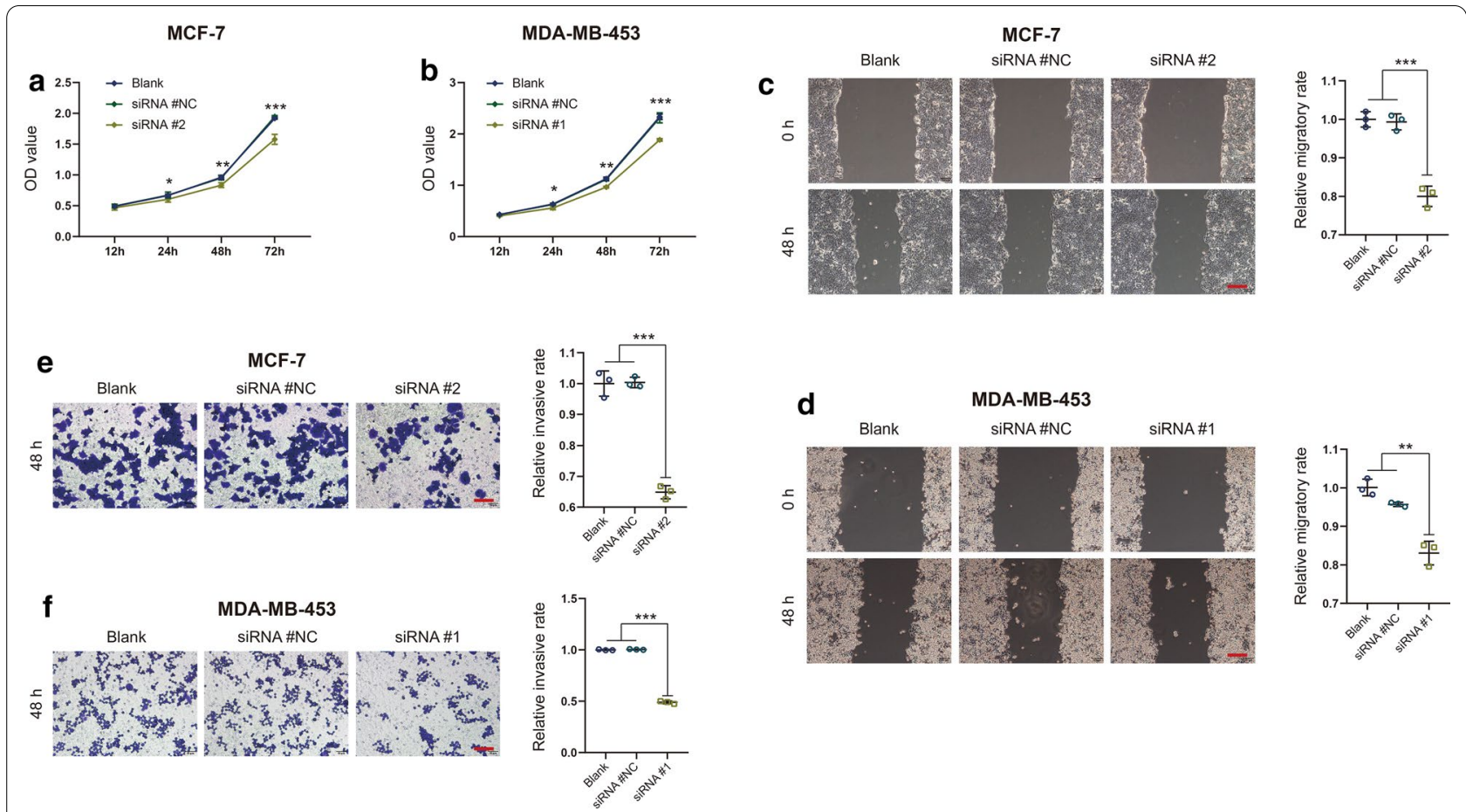

Fig. 3 Silencing UBE3C inhibits BrCa cell proliferation, migration and invasion. a, b The proliferative capacities of control and UBE3C-silenced BrCa cells were evaluated with a CCK-8 assay. UBE3C-silenced BrCa cells showed inhibited proliferative capacities. $\mathbf{c}$, d The migratory capacities of control and UBE3C-silenced BrCa cells were evaluated with a wound healing assay. UBE3C-silenced BrCa cells showed inhibited migratory capacities. $\mathbf{e}$, $\mathbf{f}$ The invasive capacities of control and UBE3C-silenced BrCa cells were evaluated with a Boyden chamber assay. UBE3C-silenced BrCa cells showed inhibited invasive capacities. ${ }^{*} P<0.05,{ }^{* *} P<0.01,{ }^{* *} P<0.001 . B a r=200 \mu m$

in cell proliferation, migration and invasion by $\mathrm{BrCa}$ cells were tested by CCK-8, wound healing and Boyden chamber assays, respectively. First, the silencing efficiency of UBE3C in BrCa cells was confirmed by qPCR, western blotting and immunofluorescence analysis (Fig. 2a-d, Additional file 2: Figure S1). Compared with corresponding control cells, UBE3C-siRNA MCF-7 and MDA-MB-453 cells showed an attenuated proliferative capacity (Fig. 3a, b). In addition, inhibition of UBE3C expression notably suppressed BrCa cell migration and invasion (Fig. 3c-f). Overall, silencing UBE3C significantly impedes $\mathrm{BrCa}$ progression in vitro.

\section{Exogenous UBE3C promotes BrCa cell proliferation, migration and invasion}

Next, we assessed the changes in cellular behavior induced by exogenous UBE3C overexpression. The transfection efficiency of UBE3C in MCF-7 and MDAMB-453 BrCa cells was confirmed by qPCR, western blotting and immunofluorescence analysis (Fig. 4a-d, Additional file 2: Figure S1). Compared with that of corresponding control cells, the proliferative capacity of UBE3C-overexpressing MCF-7 and MDA-MB-453 cells was significantly enhanced (Fig. 5a, b). In addition, exogenous expression of UBE3C remarkably increased BrCa cell migration and invasion (Fig. $5 \mathrm{c}-\mathrm{f}$ ). Overall, overexpression of UBE3C plays an oncogenic role in $\mathrm{BrCa}$ cells, which may be a potential target for $\mathrm{BrCa}$ therapy.

\section{UBE3C regulates the Wnt/ $\beta$-catenin pathway in $\mathrm{BrCa}$ cells}

The canonical Wnt pathway, namely, the Wnt/ $\beta$-catenin pathway, is one of the pathways most involved in the oncogenesis and progression of $\mathrm{BrCa}$ [15-17]. We postulated that UBE3C regulates the $\mathrm{Wnt} / \beta$-catenin signaling pathway to mediate tumor growth and metastasis in $\mathrm{BrCa}$ cells. Nuclear accumulation of $\beta$-catenin is the central event in activation of the $\mathrm{Wnt} / \beta$-catenin signaling pathway, and whether UBE3C can regulate $\beta$-catenin nuclear accumulation in $\mathrm{BrCa}$ cells was first investigated. Silencing UBE3C decreased the nuclear $\beta$-catenin level, while overexpressing UBE3C enhanced the nuclear $\beta$-catenin level in MCF-7 and MDA-MB-453 BrCa cells (Fig. 6a-d, Additional file 3: Figure S2). Besides, the results from 

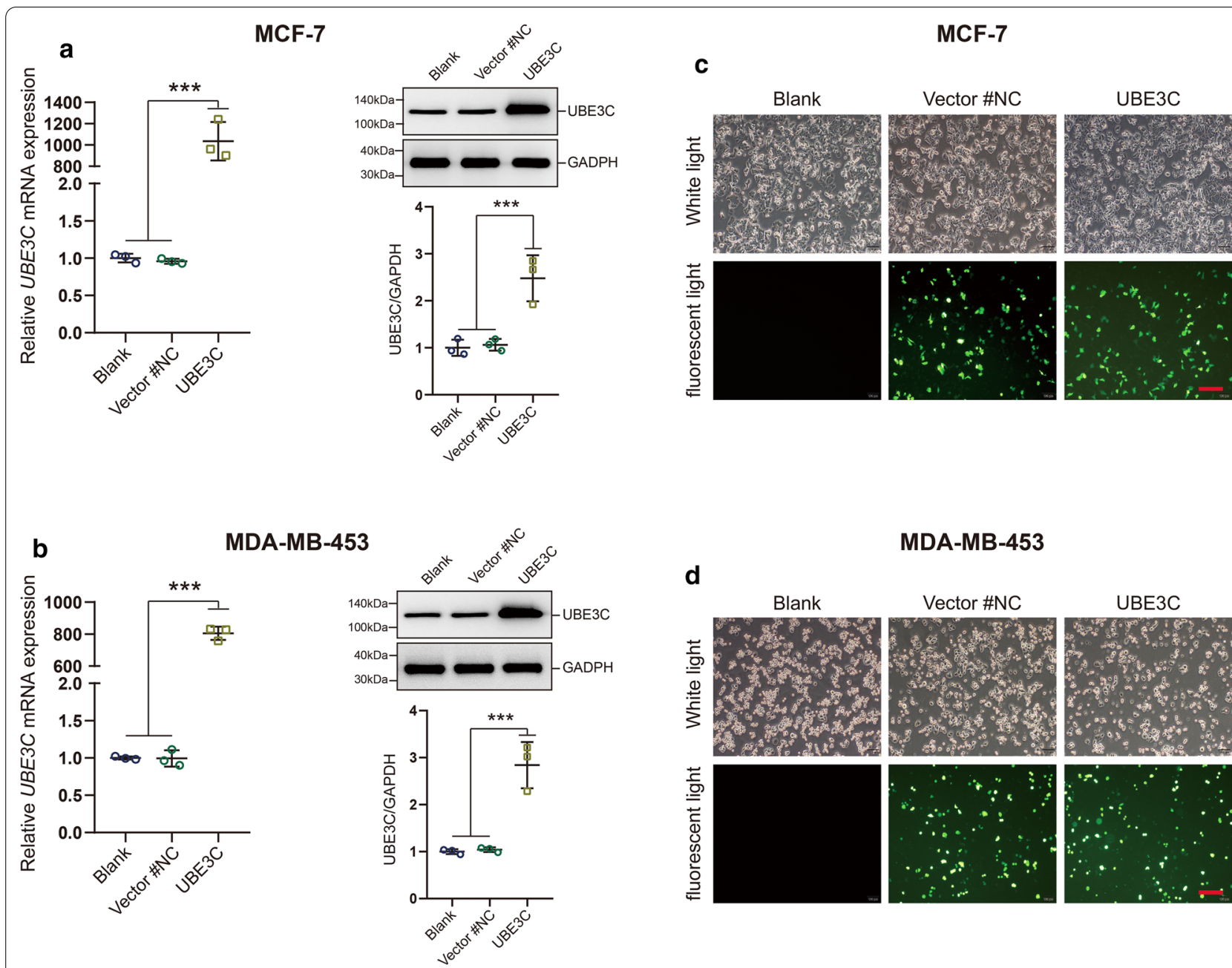

MDA-MB-453

Fig. 4 The efficiency of UBE3C overexpression in BrCa cells. $\mathbf{a}$, b The efficiency of UBE3C overexpression in BrCa cells was confirmed by qPCR and western blotting analysis. UBE3C-overexpressing vector showed satisfactory transfection efficiency. $\mathbf{c}$, $\mathbf{d}$ The efficiency of UBE3C overexpression in BrCa cells and cytomorphology was determined by microscopy and immunofluorescence analysis. Green fluorescence indicated that the cells have been successfully transfected. ${ }^{* * *} \mathrm{P}<0.001 . \mathrm{Bar}=200 \mu \mathrm{m}$

immunofluorescence show that $\mathrm{UBE} 3 \mathrm{C}$ and $\beta$-catenin is mainly located in nuclear and have positive correlation. To test whether UBE3C influences the expression levels of molecules downstream of the $\mathrm{Wnt} / \beta$-catenin signaling pathway, we next evaluated MMP9 and Cyclin D1 expression, and the results showed that silencing UBE3C inhibited their expression and that exogenous UBE3C had the opposite effects (Fig. 7a, b, Additional file 4: Figure S3). Taken together, these data suggest that UBE3C can regulate $\beta$-catenin nuclear accumulation in $\mathrm{BrCa}$ cells, which may be the critical mechanism promoting $\mathrm{BrCa}$ progression.

\section{Discussion}

Ubiquitination has been a research hotspot in recent years. Ubiquitin ligase E3 directly mediates the specific binding of ubiquitin to substrates and may be involved in the regulation of multiple signaling pathways, which is essential for critical cellular processes [18-20]. Ubiquitin ligases are mainly classified into two types: homologous to E6AP C-terminus (HECT) and really interesting new gene (RING) [21]. UBE3C is a member of the HECT family, and proteins in this family contain two main protein domains: an IQ motif and a HECT domain [21]. The IQ motif mediates substrate targeting, and the HECT domain binds to ubiquitin-conjugating enzymes (E2) and mediates ubiquitin conjugation to the target substrate 


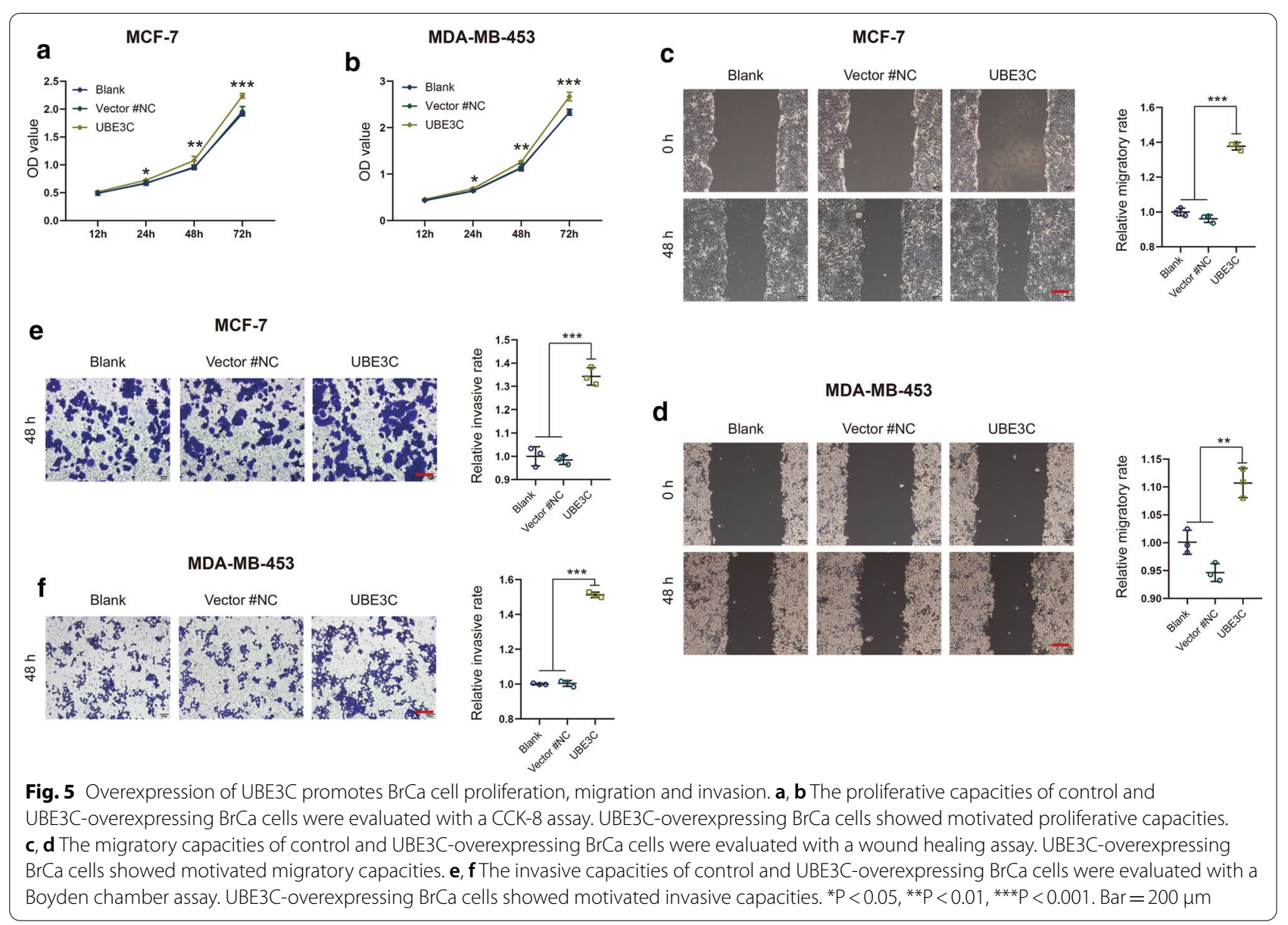

[22]. Therefore, the HECT domain of UBE3C provides E3 ligase catalytic activity.

E3 ligases play critical roles in the regulation of multiple biological signals via selectively binding to their specific protein substrates [18, 23]. Dysregulation of the HECT E3 ligase contributes to various pathological disorders, including human cancers [24-26]. E6AP, the first identified member of the HECT E3 family [27], targets the tumor suppressor TP53 through ubiquitinmediated proteolysis, which enhances the degradation of the TP53 protein and leads to the progression of various human cancers [24]. UBE3C, another member of the HECT E3 family, has been reported to play a critical role in multiple cancers [8-10]. However, there are currently no reports on the association between UBE3C and the progression of $\mathrm{BrCa}$.

In this study, we found that UBE3C expression was significantly upregulated in $\mathrm{BrCa}$ tissues compared with adjacent breast tissues. In addition, high nuclear UBE3C expression was associated with an advanced TNM stage and a poor survival status. In addition, we confirmed that BrCa patients with high nuclear UBE3C expression in tissues showed worse OS than those with low nuclear UBE3C expression but that high cytoplasmic UBE3C expression did not produce a similar effect. Taken together, these results suggest that nuclear UBE3C protein expression may be a novel diagnostic and prognostic biomarker for $\mathrm{BrCa}$.

In addition to the diagnostic and prognostic value of UBE3C, several scholars have found that targeting $\mathrm{UBE} 3 \mathrm{C}$ is a promising therapeutic strategy to limit cancer progression $[12,28]$. In this study, we confirmed that targeting UBE3C expression using siRNA significantly inhibited the proliferation, migration and invasion of BrCa MCF-7 and MDA-MB-453 cells. However, exogenous overexpression of UBE3C endowed BrCa MCF-7 and MDA-MB-453 cells with a stronger malignant potential.

The Wnt/ $\beta$-catenin signaling pathway has been reported to be related to the oncogenesis and progression of multiple kinds of human cancers [15-17]. $\beta$-catenin is considered a positive regulator of this pathway that 


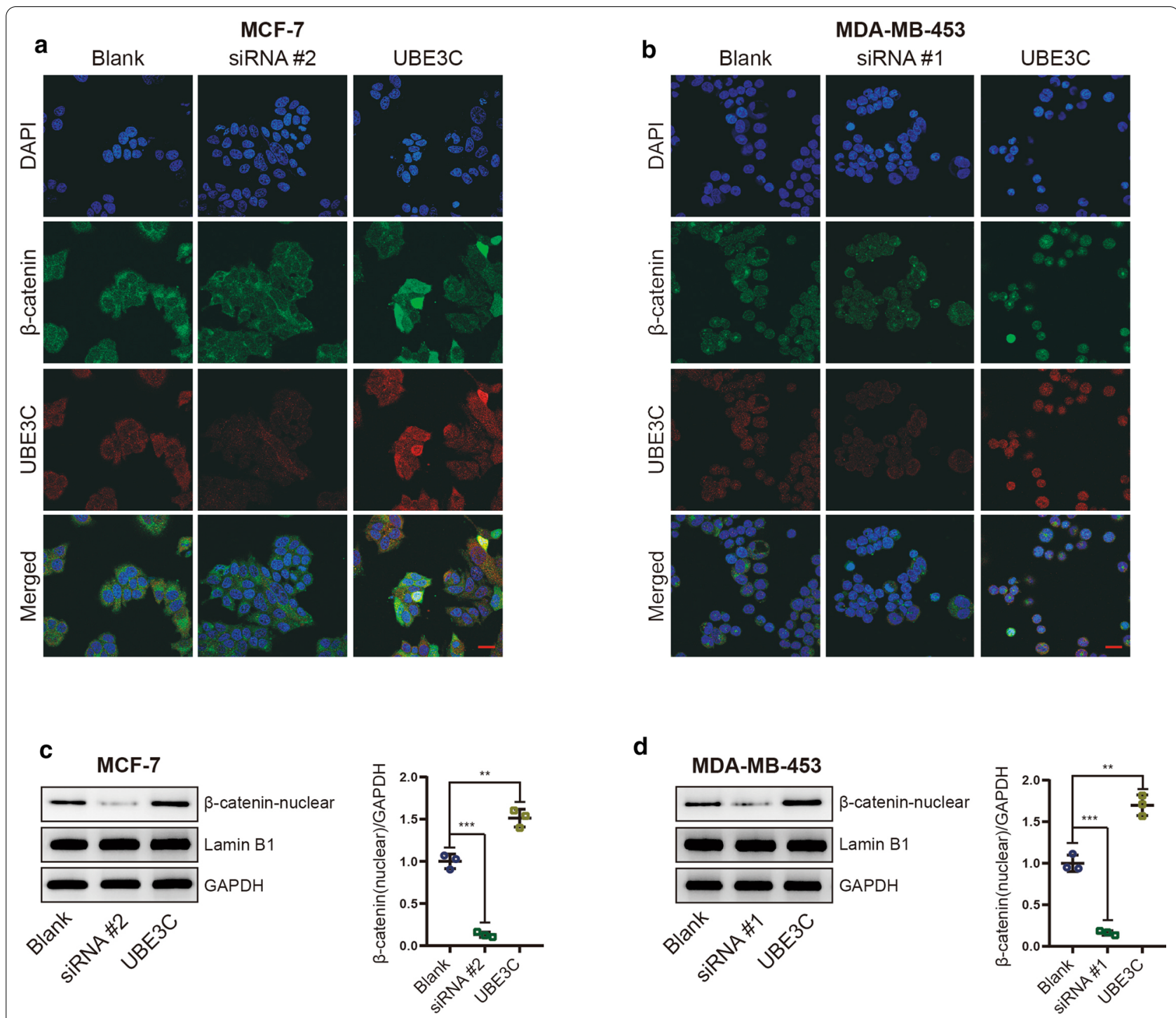

Fig. 6 UBE3C promotes the nuclear accumulation of $\beta$-catenin. $\mathbf{a}, \mathbf{b}$ Immunofluorescence staining shows that silencing or overexpressing UBE3C in BrCa cells inhibited or increased the nuclear accumulation of $\beta$-catenin, respectively. Blue: DAPI, green: $\beta$-catenin, red: UBE3C. Bar $=20 \mu \mathrm{m}$. $\mathbf{c}, \mathbf{d}$ The expression levels of total and nuclear $\beta$-catenin in UBE3C-silenced and/or UBE3C-overexpressing BrCa cells was evaluated by western blotting. UBE3C-silenced BrCa cells showed low nuclear $\beta$-catenin level, but UBE3C-overexpressing BrCa cells were opposite. ${ }^{* *} \mathrm{P}<0.01,{ }^{* * *} \mathrm{P}<0.001$

functions by promoting the transcription of target genes [29]. Some target genes, such as MMP9 and cyclin D1, are involved in oncogenesis, regulating cancer proliferation and metastasis [30,31]. To the best of our knowledge, there is no research on the association between UBE3C and regulation of the $\mathrm{Wnt} / \beta$-catenin pathway in BrCa. In this study, we found that silencing UBE3C decreased the nuclear $\beta$-catenin level, while overexpressing UBE3C enhanced the nuclear $\beta$-catenin level in MCF-7 and MDA-MB-453 BrCa cells. In addition, silencing UBE3C inhibited MMP9 and Cyclin D1 expression, and exogenous UBE3C had the opposite effects. Altogether, these results demonstrate that UBE3C may be associated with cell proliferation, migration and invasion in $\mathrm{BrCa}$ by mediating the $\mathrm{Wnt} / \beta$-catenin signaling pathway. In gastric cancer, Zhang et al. demonstrated that UBE3C promotes gastric cancer progression through activating the $\beta$-catenin signaling via degradation of AXIN1 [32], which explained the potential mechanism of UBE3C regulating $W n t / \beta$-catenin signaling pathway. 

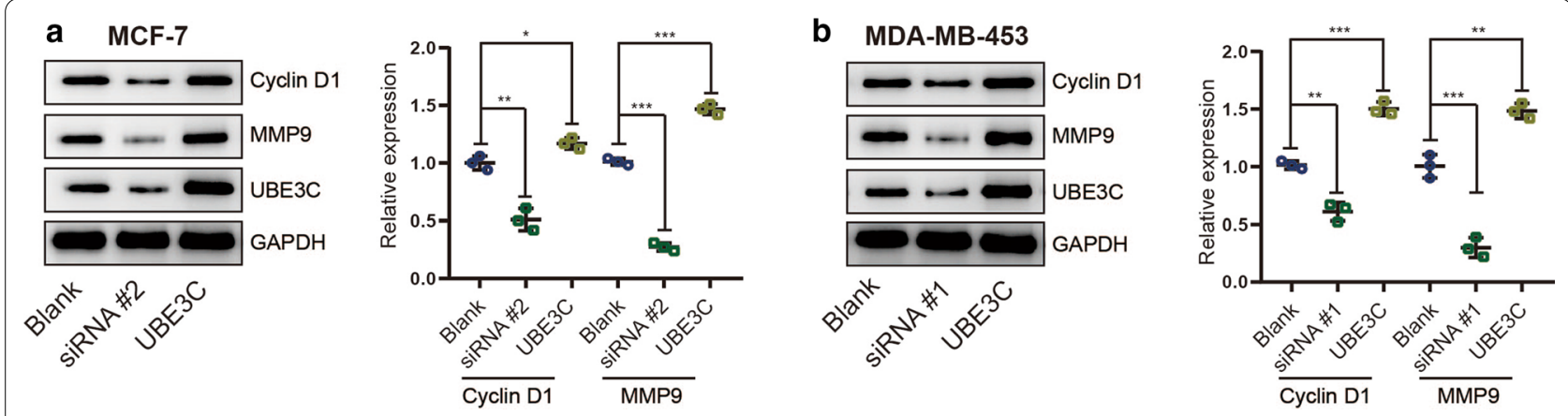

Fig. 7 UBE3C regulates expression of Cyclin D1 and MMP9 in BrCa cell. $\mathbf{a}$, b The expression levels of UBE3C, Cyclin D1 and MMP9 in UBE3C-silenced and/or UBE3C-overexpressing BrCa cells were evaluated by western blotting. UBE3C-silenced BrCa cells showed low Cyclin D1 and MMP9 levels, but UBE3C-overexpressing BrCa cells were opposite. ${ }^{*} \mathrm{P}<0.05$, ${ }^{* *} \mathrm{P}<0.01$, ${ }^{* * *} \mathrm{P}<0.001$

\section{Conclusion}

Overall, the present study evaluated the possibility of using UBE3C as a potential biomarker for disease diagnosis, as well as identifying UBE3C as a promising indicator of the prognosis of patients with $\mathrm{BrCa}$, for the first time. In addition, in agreement with clinical findings, in vitro cell experiments confirmed that UBE3C enhances $\mathrm{BrCa}$ cell proliferation, migration and invasion by mediating the $\mathrm{Wnt} / \beta$-catenin signaling pathway. Overall, UBE3C plays critical roles in $\mathrm{BrCa}$ carcinogenesis and progression, and UBE3C may be a novel target for $\mathrm{BrCa}$ therapy.

\section{Supplementary Information}

The online version contains supplementary material available at https://doi. org/10.1186/s12935-020-01733-7.

Additional file 1: Table S1. Association between UBE3C expression and patients' clinical parameters in $\mathrm{BrCa}$.

Additional file 2: Figure S1. The original image for western blotting in Fig. 2 and Fig. 4.

Additional file 3: Figure S2. The original image for western blotting in Fig. 6.

Additional file 4: Figure S3. The original image for western blotting in Fig. 7.

\section{Abbreviations}

BrCa: Breast cancer; UBE3C: Ubiquitin protein ligase E3C; TMA: Tissue microarray; IHC: Immunohistochemistry; IRS: Immunoreactivity score; FBS: Fetal bovine serum; CCK-8: Cell Counting Kit-8; TNM: Tumor node metastasis classification; OS: Overall survival; siRNA: Small interfering RNA; RING: Really interesting new gene; HECT: Homologous to E6AP C-terminus; E2: Ubiquitinconjugating enzymes.

\section{Acknowledgments}

Not applicable.

\section{Authors' contributions}

YZ conceived the study and participated in the study design, performance, coordination and manuscript writing. $\mathrm{CH}, \mathrm{SZ}$, and TW carried out the assays and analysis. YZ revised the manuscript. All authors reviewed the final manuscript. All authors read and approved the final manuscript.

\section{Funding}

This work was funded by the Wuxi Science and Technology Bureau Project (No. CSE31N1720), the Jiangsu Province Youth Medical Talent Fund Project (No. QNRC2016166), the Jiangsu Provincial Six Talent Peaks Project (No. YY-124) and Jiangsu Provincial Maternal and child Health Research Project (F202034). The funding bodies had no role in the study design; the collection, analysis, or interpretation of data; writing the manuscript; or the decision to publish.

Availability of data and materials

All data are included in the article.

Ethics approval and consent to participate

Not applicable.

Consent for publication

Not applicable.

Competing interests

The authors declare no conflicts of interest.

\section{Author details}

${ }^{1}$ Department of Oncology, Wuxi Maternal and Child Health Hospital Affiliated to Nanjing Medical University, Wuxi 214023, China. ${ }^{2}$ Department of Gynecology and Obstetrics, Wuxi Maternal and Child Health Hospital Affiliated to Nanjing Medical University, No. 48, Huaishu Road, Wuxi 214023, China.

Received: 29 September 2020 Accepted: 21 December 2020

Published online: 06 January 2021

\section{References}

1. Siegel RL, Miller KD, Jemal A. Cancer statistics, 2019. CA Cancer J Clin. 2019;69(1):7-34.

2. Dean A. Primary breast cancer: risk factors, diagnosis and management. Nurs Stand. 2008;22(40):47-55 (quiz 58, 60).

3. Peart O. Breast intervention and breast cancer treatment options. RadiolTechnol. 2015;86(5):535M-558M (quiz 559-562).

4. Shen T, Gao C, Zhang K, Siegal GP, Wei S. Prognostic outcomes in advanced breast cancer: the metastasis-free interval is important. Hum Pathol. 2017;70:70-6.

5. Mei J, Hao L, Liu X, Sun G, Xu R, Wang H, Liu C. Comprehensive analysis of peroxiredoxins expression profiles and prognostic values in breast cancer Biomark Res. 2019;7:16

6. Wu S, Guo X, Zhou J, Zhu X, Chen H, Zhang K, Lu Y, Chen Y. High expression of UNC5B enhances tumor proliferation, increases metastasis, and worsens prognosis in breast cancer. Aging (Albany NY). 2020;12:17079.

7. Kuo CL, Goldberg AL. Ubiquitinated proteins promote the association of proteasomes with the deubiquitinating enzyme Usp14 and the ubiquitin ligase Ube3c. Proc Natl Acad Sci U S A. 2017;114(17):E3404-13. 
8. Tang L, Yi XM, Chen J, Chen FJ, Lou W, Gao YL, Zhou J, Su LN, Xu X, Lu JQ, et al. Ubiquitin ligase UBE3C promotes melanoma progression by increasing epithelial-mesenchymal transition in melanoma cells. Oncotarget. 2016;7(13):15738-46.

9. Wen JL, Wen XF, Li RB, Jin YC, Wang XL, Zhou L, Chen HX. UBE3C promotes growth and metastasis of renal cell carcinoma via activating Wnt/ beta-catenin pathway. PLoS ONE. 2015;10(2):e0115622.

10. Gu J, Mao W, Ren W, Xu F, Zhu Q, Lu C, Lin Z, Zhang Z, Chu Y, Liu R, et al. Ubiquitin-protein ligase E3C maintains non-small-cell lung cancer stemness by targeting AHNAK-p53 complex. Cancer Lett. 2019:443:125-34.

11. Okada M, Ohtake F, Nishikawa H, Wu W, Saeki Y, Takana K, Ohta T. Liganded ERalpha stimulates the E3 ubiquitin ligase activity of UBE3C to facilitate cell proliferation. MolEndocrinol. 2015;29(11):1646-57.

12. Xiong J, Wei $B$, Ye $Q$, Liu W. MiR-30a-5p/UBE3C axis regulates breast cancer cell proliferation and migration. Biochem Biophys Res Commun. 2019;516(3):1013-8.

13. Mei J, Yan T, Huang Y, Xia T, Chang F, Shen S, Hao L, Chen Y, Wang Z, Jiang $X$, et al. A DAAM1 3'-UTR SNP mutation regulates breast cancer metastasis through affecting miR-208a-5p-DAAM1-RhoA axis. Cancer Cell Int. 2019;19:55.

14. Mei J, Xu B, Hao L, Xiao Z, Liu Y, Yan T, Zhu Y. Overexpressed DAAM1 correlates with metastasis and predicts poor prognosis in breast cancer. Pathol Res Pract. 2020;216(3):152736.

15. Wang Z, Li Z, Wu Q, Li C, Li J, Zhang Y, Wang C, Sun S, Sun S. DNER promotes epithelial-mesenchymal transition and prevents chemosensitivity through the Wnt/beta-catenin pathway in breast cancer. Cell Death Dis. 2020;11(8):642.

16. Shahzad N, Munir T, Javed M, Tasneem F, Aslam B, Ali M, Mutahir Z, Akhtar Ali M, Umer M, Ahmad M, et al. SHISA3, an antagonist of the Wnt/betacatenin signaling, is epigenetically silenced and its ectopic expression suppresses growth in breast cancer. PLoS ONE. 2020;15(7):e0236192.

17. Rahmani F, TadayyonTabrizi A, Hashemian P, Alijannejad S, Rahdar HA, Ferns GA, Hassanian SM, Shahidsales S, Avan A. Role of regulatory miRNAs of the Wnt/ beta-catenin signaling pathway in tumorigenesis of breast cancer. Gene. 2020;754:144892.

18 Park HB, Kim JW, Baek KH. Regulation of Wnt signaling through ubiquitination and deubiquitination in cancers. Int J Mol Sci. 2020;21(11):3904.

19. Deng L, Meng T, Chen L, Wei W, Wang P. The role of ubiquitination in tumorigenesis and targeted drug discovery. Signal Transduct Target Ther. 2020;5(1):11.

20. Perez-Benavente B, Nasresfahani AF, Farras R. Ubiquitin-regulated cell proliferation and cancer. AdvExp Med Biol. 2020;1233:3-28.
21. Metzger MB, Hristova VA, Weissman AM. HECT and RING finger families of E3 ubiquitin ligases at a glance. J Cell Sci. 2012;125(Pt 3):531-7.

22. Zheng N, Shabek N. Ubiquitin ligases: structure, function, and regulation. Annu Rev Biochem. 2017:86:129-57.

23. Zhou X, Li Y, Wang W, Wang S, Hou J, Zhang A, Lv B, Gao C, Yan Z, Pang $D$, et al. Regulation of hippo/YAP signaling and Esophageal squamous carcinoma progression by an E3 ubiquitin ligase PARK2. Theranostics. 2020;10(21):9443-57.

24 Owais A, Mishra RK, Kiyokawa H. The HECTE3 ligase E6AP/UBE3A as a therapeutic target in cancer and neurological disorders. Cancers (Basel). 2020;12(8):2108.

25. Gong X, Du D, Deng Y, Zhou Y, Sun L, Yuan S. The structure and regulation of the E3 ubiquitin ligase HUWE1 and its biological functions in cancer. Invest New Drugs. 2020;38(2):515-24.

26. Bernassola F, Chillemi G, Melino G. HECT-type E3 ubiquitin ligases in cancer. Trends Biochem Sci. 2019;44(12):1057-75.

27. Bernassola F, Karin M, Ciechanover A, Melino G. TheHECT family of E3 ubiquitin ligases: multiple players in cancer development. Cancer Cell. 2008;14(1):10-21.

28. Tao J, Liu Z, Wang Y, Wang L, Yao B, Li Q, Wang C, Tu K, Liu Q. MiR-542-3p inhibits metastasis and epithelial-mesenchymal transition of hepatocellular carcinoma by targeting UBE3C. Biomed Pharmacother. 2017;93:420-8.

29. Wen X, Wu Y, Awadasseid A, Tanaka Y, Zhang W. New Advances in canonical Wnt/beta-catenin signaling in cancer. CancerManag Res. 2020;12:6987-98.

30. Lan S, Zheng X, Hu P, Xing X, Ke K, Wang F, Cheng N, Zhuang Q, Liu X, Liu $J$, et al. Moesin facilitates metastasis of hepatocellular carcinoma cells by improving invadopodia formation and activating beta-catenin/MMP9 axis. Biochem Biophys Res Commun. 2020;524(4):861-8.

31. Zhang GC, Yu XN, Sun JL, Xiong J, Yang YJ, Jiang XM, Zhu JM. UBE2M promotes cell proliferation via the beta-catenin/cyclin D1signaling in hepatocellular carcinoma. Aging (Albany NY). 2020;12(3):2373-92.

32. Zhang $Y, X u$ J, Fu H, Wei Z, Yang D, Yan R. UBE3C promotes proliferation and inhibits apoptosis by activating the beta-catenin signaling via degradation of AXIN1 in gastric cancer. Carcinogenesis. 2020. https://doi. org/10.1093/carcin/bgaa098.

\section{Publisher's Note}

Springer Nature remains neutral with regard to jurisdictional claims in published maps and institutional affiliations.
Ready to submit your research? Choose BMC and benefit from:

- fast, convenient online submission

- thorough peer review by experienced researchers in your field

- rapid publication on acceptance

- support for research data, including large and complex data types

- gold Open Access which fosters wider collaboration and increased citations

- maximum visibility for your research: over $100 \mathrm{M}$ website views per year

At BMC, research is always in progress.

Learn more biomedcentral.com/submissions 